\title{
LA EXPERIENCIA PSICOSOCIAL DE PADRES DE HIJOS CON LEUCEMIA EN UN HOSPITAL PÚBLICO DE LA CIUDAD DE SAN LUIS POTOSí
}

\section{THE PSYCHOSOCIAL EXPERIENCE OF PARENTS OF CHILDREN WITH LEUKEMIA IN A PUBLIC HOSPITAL IN THE CITY OF SAN LUIS POTOSI}

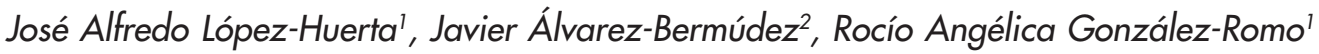 \\ ' Facultad de Psicología de la Universidad. Autónoma de San Luis Potosí. México. \\ ${ }^{2}$ Facultad de Psicología de la Universidad. Autónoma de Nuevo León. México.
}

Resumen

El objetivo general de la investigación fue identificar la experiencia psicosocial en el contexto de enfermedad en padres-cuidadores primarios de hijos con leucemia. Esta investigación es de tipo cualitativo, se aplicaron como técnicas de obtención de información una entrevista semi-estructurada a los padres y se realizó observación no participante en la sala de espera del Hospital. El tipo de análisis de información que realizamos fue análisis de contenido. Una de las categorías principales que identificamos como influyentes en dicha experiencia, es la pobreza de los sujetos y sus comunidades de procedencia. Esta característica socioeconómica se convierte en criterio de identidad social para los sujetos quienes se saben pertenecientes a un grupo de personas desfavorecidas, lo que promueve en gran medida actividades de afrontamiento ante las necesidades que por su condición deben dar solución. La falta de recursos es una fuente de ansiedad para el sujeto al enfrentar el desafío de encontrar recursos para solventar los gastos derivados del tratamiento de la enfermedad. Estos resultados muestran en gran medida la inequidad que existe con relación al acceso a la salud pública derivada del lugar y condición en que vive la persona.

Palabras clave: Identidad social, Leucemia, cuidadores primarios, representaciones sociales, condiciones sociales, inequidad.
Abstract

The general research objective was to identify the psychosocial experiences in the context of disease in parents-primary caregivers of children with Leukemia. Applied semi-structured interview to parents and non-participant observation in the hospital waiting room. One of the main categories identified as influential in this experience, is the poverty of individuals and their communities of origin. Lack of resources is a source of anxiety for the individual to face the challenge of finding resources to cover the costs of treating the disease. These results show largely inequity that exists with regard to access to public health arising from the location and condition in which the individual lives.

Keywords: Social identity, Leukemia, primary caregivers, social representations, social conditions, inequity.

\section{Correspondencia:}




\section{INTRODUCCIÓN}

El cáncer, en general, es una de las enfermedades con más altos índices de morbilidad y mortalidad en el mundo, de acuerdo a la Organización Mundial de la Salud (OMS), en el año 2005 el cáncer fue la segunda causa de muerte dentro del grupo de enfermedades no transmisibles, provocando el $13 \%$ de muertes de un total de 58 millones, característica que se espera se incrementará en el futuro ${ }^{(1,2)}$.

En países como Inglaterra, Irlanda, Francia, Australia y Dinamarca la leucemia es el tipo de cáncer de mayor incidencia en el grupo de edad de 0 a 14 años $^{(3)}$.

En México el cáncer es la tercera causa de muerte. En el año 2006, 63.888 personas fallecieron por tumores malignos, lo que representó el 12,9\% del total de las defunciones registradas. En el grupo de edad de 5 a 14 años, el cáncer es la segunda causa de muerte. La leucemia provocó el $52,2 \%$ de los fallecimientos ocurridos en este grupo de edad y los tumores malignos el 16,3\% de los decesos. En el caso específico del estado de San Luís Potosí, lugar en que se realizó la investigación, la leucemia y los tumores malignos se mantienen como la segunda causa de mortalidad en el grupo de edad de 5 a 14 años $^{(5,6)}$.

Diversas acciones para atender el cáncer han permitido un incremento importante en la expectativa de vida de los pacientes, a pesar de lo cual se tienen costos emocionales importantes derivados del riesgo latente de alguna recaída ${ }^{(7)}$.

Las condiciones estructurales en nuestro país para la atención del cáncer son insuficientes, por ejemplo el gasto per cápita en México en el año 2009 fue de $\$ 514,801958$ dólares norteamericanos, en Argentina fue de $\$ 695,46186$, en Brasil de $\$ 734,051382$, en Colombia de $\$ 323,010813$, en Uruguay de $\$ 698,163292$, el promedio en los países miembros de la OCDE fue de $\$ 3973,50217$, de tal forma que en compa- ración con países en condiciones similares de desarrollo y de los países miembros de la OCDE la inversión en atención oncológica es baja ${ }^{(8)}$.

Con relación a la formación en recursos humanos para la salud, egresan al año en el país aproximadamente 20 mil médicos, de los cuales el 5\% sigue una especialidad y de éstos menos del $10 \%$ la ejerce, los pocos especialistas en oncología pediátrica que egresan no cuentan con la infraestructura necesaria para Ilevar a cabo sus actividades. En el año 2008 atendiendo a esta problemática el Senado de la República aprobó el dictamen con punto de acuerdo por el que se exhorta al titular del Poder Ejecutivo Federal a fortalecer las medidas para atender y prevenir el cáncer infanti $\left.\right|^{(9)}$.

Los diversos estudios desde la psicología y otras áreas, han mostrado que cuando uno ha tenido la experiencia de vivir de manera directa o indirecta una enfermedad crónica y/o terminal, ésta afecta todas las áreas de la vida, no sólo del paciente sino también de sus familiares cercanos $^{(10,13)}$ y cuando el paciente es un hijo, el impacto emocional es mayor al que se puede presentar ante la enfermedad de otro miembro de la familia ${ }^{(14)}$ y los niveles de estrés tienden a ser más altos conforme es menos la edad del paciente ${ }^{(15)}$.

Desde el inicio del proceso de enfermedad, los pacientes menores de edad dependen de los cuidados que proveen los adultos. Cuando se elige el cuidador primario, es generalmente la madre quien es asignada a tal rol, el cual es construido cultural y socialmente, ya que se considera como la persona idónea para proporcionar los cuidados ${ }^{(16)}$.

En el momento del diagnóstico de cáncer, la familia pasa por un periodo de crisis. Surgen una serie de emociones que hay que enfrentar y se deben tomar decisiones rápidas en un contexto de ansiedad e incertidumbre, derivado de la ignorancia 
respecto a la enfermedad y a las características del tratamiento(17).

Los padres han de afrontar problemas que agravan la vivencia de la enfermedad, situaciones nuevas para las cuales no están preparados, acciones tales como relacionarse con el personal médico, trasladarse a lugares desconocidos hasta ese momento como hospitales y consultorios, la separación de familiares y amigos así como cambios en el rol social, entre otros ${ }^{(18)}$. La experiencia difícil de que diagnostiquen a un hijo con cáncer, se ve agravada cuando los recursos son pocos tanto para obtener primeramente el diagnóstico como posteriormente iniciar y dar seguimiento al tratamiento.

Dentro de los cambios que se presentan a corto, mediano y largo plazo en el área emocional tanto en pacientes como en familiares, se han encontrado altos índices de estrés, depresión, temor y preocupación debido a las expectativas tan diversas que genera la enfermedad, entre ellas la de muerte y a factores socioeconómicos como los gastos derivados del tratamiento de la enfermedad y del cuidado del paciente ${ }^{(19,21)}$, específicamente en las madres de niños con cáncer se han identificado altos índices de depresión y uso de estrategias de afrontamiento centrado en la emoción, variables que han sido asociadas en algunos estudios, con problemas conductuales del niño ${ }^{(22)}$.

Se ha encontrado en algunas investigaciones que los padres presentan en un inicio emociones negativas las cuales posteriormente tienden a tornarse positivas ${ }^{(23,25)}$. Sin embargo a pesar de este cambio emocional también se ha encontrado en las diferentes fases de la enfermedad, síntomas de estrés postraumático tanto en los pacientes infantiles como en sus padres, siendo estos últimos quienes presentan más síntomas de estrés que los pacientes ${ }^{(26,27)}$. En este mismo sentido la carga emocional del paciente tiene un amplio rango, que va de dar narraciones cargadas de sentimientos y significado, hasta narraciones en las que hay una ausencia significativa en referencia a tales dimensiones ${ }^{(28)}$.

A pesar del interés en el cuidado del hijo enfermo algunas veces esta actividad afecta de forma negativa en los padres, al ver frustrados planes personales y alterar su vida cotidiana como la disminución de sus horas de sueño. Asimismo, los efectos secundarios del tratamiento como el dolor, la fatiga y los trastornos de sueño, se han asociado también con una disminución en la calidad de vida de los padres ${ }^{(29)}$.

En el caso específico de las madres se han encontrado correlaciones positivas entre la calidad de vida de la madre y la calidad de vida del hijo por lo que una baja calidad de vida de la madre se relaciona con una baja calidad de vida del hijo en indicadores como: salud física, emocional y social; después de 2 a 5 meses de haber recibido el diagnóstico ${ }^{(30)}$.

En el caso de los padres se ha encontrado que cuando perciben en sí mismos altos niveles de bienestar, igualmente perciben que la salud de sus hijos es mejor ${ }^{(31)}$.

Desde una perspectiva psicosocial, dimensión que en los últimos tiempos se ha mostrado relevante, tenemos un área de oportunidad amplia tanto para la investigación como para el desarrollo de estrategias de intervención. Un área de trabajo es la vivencia de los cuidadores primarios y de los pacientes con relación a la enfermedad en un contexto de carencia de recursos, pues sin duda esta característica afecta de forma negativa la experiencia ya de por sí difícil de tener un hijo con Leucemia.

El fundamento teórico para esta investigación es la Teoría de Representaciones Sociales (TRS), la cual surge en gran medida del interés por identificar como es que el conocimiento científico o las teorías científicas son reproducidas a pesar de no te- 
ner antecedentes o conocimientos previos y que sin embargo a pesar de esto las ideas circulan, se transmiten independientemente de la explicación o sustento científico original, estas explicaciones conforman las teorías de sentido común cotidiano en la vida diaria de los sujetos ${ }^{(32,33)}$.

Las representaciones tienen una realidad psíquica relacionada con los afectos y los pensamientos, así como una realidad externa relacionada con la colectividad y los grupos. En estas dos realidades descansa el aspecto dinámico de una representación social, es decir, las personas asimilan y modifican la representación o representaciones obtenidas de su medio social y las reintegran modificadas al mismo(33), por tanto el conocimiento de sentido común no puede ser incorrecto y malo si es útil sirviendo como guía en la vida cotidiana de las personas ${ }^{(32,34)}$.

Se han propuesto diversas definiciones de lo que es una representación social, en este trabajo a manera de definición consideramos la representación social como una estructura cognitiva, afectiva, evaluativa y operativa de fenómenos socialmente relevantes de los cuales los individuos están conscientes y los cuales son compartidos por otros miembros del grupo social( $^{(35)}$.

Esta conceptualización se asocia estrechamente con una perspectiva procesual e interpretativa en el contexto de la Teoría de las Representaciones Sociales y entre sus características encontramos las siguientes: tiende a la interdisciplinariedad, al uso de métodos cualitativos, a la construcción social de la realidad y a temas como la sociogénesis y el afrontamiento simbólico individual y colectivo ${ }^{(36,37)}$.

En la construcción de una representación social existen dos proceso básicos, el primero de ellos es el anclaje y se refiere al enraizamiento social de la representación y de su objeto, lo social se traduce en: a) significado y b) utili- dad que le son conferidos, c) integración cognitiva del objeto representado dentro del sistema de pensamiento preexistente y a las transformaciones derivadas de este sistema, tanto de una parte como de otra. En general el anclaje es clasificar y nombrar algo siempre en función de nuestros antecedentes $^{(37)}$. El anclaje articula tres funciones básicas de la representación: la cognitiva, de interpretación de la realidad, y de la orientación de las conductas y las relaciones sociales ${ }^{(38)}$ :

El segundo proceso es la objetivación. Este proceso consiste básicamente en la materialización de lo abstracto, proporciona una textura material a las ideas, las objetiva, materializa la palabra, hace corresponder cosas con palabras. La objetivación es uno de los procesos fundamentales de una representación social. Inicialmente se planteaban dos fases básicas en el proceso de objetivar. La naturalización y la clasificación. Por medio de la naturalización los elementos del pensamiento pasan a ser parte de la realidad del sujeto, la clasificación actúa colocando y organizando las partes del ambiente a un orden preexistente $^{(39)}$.

En este proceso de construcción social de una representación la identidad social tiene un papel determinante, pues la identificación del sujeto con el grupo es un requisito para la construcción de sus representaciones pues estas se nutren de del grupo o grupos con los que se identifica $^{(40)}$, es importante considerar que muchos discursos entre las personas no están restringidos local o físicamente a contextos determinados, sino que están fundados en contextos simbólicos, es decir, tienen antecedentes en común aún y cuando no se comuniquen cotidiana o físicamente para compartir significados ${ }^{(41,42)}$. Por este motivo nuestra pregunta de investigación base de este trabajo fue ¿Cómo vive el padre-cuidador primario la situación de enfermedad de un hijo con Leucemia? 


\section{MÉTODO}

La investigación se realizó en el Hospital Central Dr. Ignacio Morones Prieto de la ciudad de San Luis Potosí, México en un periodo de tiempo comprendido entre los años 2007 y 2009. El primer contacto para el acceso al Hospital se estableció con el Oncólogo Pediatra a quien se le planteó el interés de hacer la investigación. El oncólogo nos envió con el responsable del área de pediatría quién decide si es de interés para el Hospital la investigación para la cual se solicita el permiso. Con su aprobación se acude ante el Comité de Investigación y Ética del Hospital para someter a revisión el proyecto. Así obtuvimos la aprobación al mismo con el número de registro de protocolo 34-07.

Esta investigación es de tipo cualitativo porque buscamos conocer las cualidades de los participantes, de sus procesos de construcción de los significados ante objetos o situaciones determinadas, en este caso la experiencia de los padres en el contexto de la enfermedad de Leucemia en un hijo y en un contexto socioeconómico desfavorable, de igual forma fue relevante para el estudio conocer la experiencia de la persona, el cómo, cuándo y en donde, es decir, el contexto del participante, dimensiones que desde otro acercamiento en investigación nos resultan difíciles de identificar ${ }^{(43)}$.

Para el diseño de la investigación procedimos basándonos en las siguientes 3 fases generales propuestas por Janesick (2003:52), 1. Preparación, Decisiones de diseño, 2. Exploración y prueba, y por último 3. Iluminación y formulación.

En la fase uno se realizaron las siguientes acciones: Diseño de preguntas de investigación, Selección de lugar y casos, Criterios de Inclusión, Negociación para el acceso al campo de trabajo, Selección de estrategias de investigación. Para el diseño de la entrevista partimos de preguntas generales relacionadas con la experiencia de tener un hijo con cáncer, acentuado intereses prácticos como las características del proceso de diagnóstico y como y de donde obtenía recursos para atender los gastos derivados de la enfermedad, de igual forma se determinó el lugar en el que se haría la observación y se definieron los elementos a observar, que fueron básicamente transcribir conversaciones cuyo contenido se relacionara con la vivencia del cáncer. En la segunda fase se diseño la entrevista semiestandarizada, una guía de observación, se realizó el análisis y reducción de datos y finalmente la redacción del documento.

A partir de la Teoría de Representaciones Sociales lo que buscamos conocer son las teorías de sentido común que los participantes tienen respecto a su experiencia ${ }^{(44,45)}$. Las técnicas utilizadas son la entrevista semiestandarizada y el método de interpretación fue el análisis de contenido ${ }^{(46-48)}$. Aunque no hay un proceso establecido para el análisis de contenido, se siguieron las fases de preparación, organización de datos y redacción del infor$\mathrm{me}^{(47-49)}$. En el caso específico del análisis, la confiabilidad se trabajo con calificadores pares para establecer la codificación de respuestas ${ }^{(50)}$.

\section{Sujetos}

Los criterios de inclusión fueron:

- Cuidadores primarios del paciente. Considerando como cuidador primario al responsable principal del cuidado del paciente.

- Que sus pacientes tuvieran edades comprendidas entre los 10 y 15 años.

- Que acudieran a consulta al servicio ambulatorio de oncología pediátrica del Hospital Central Dr. Ignacio Morones Prieto.

- Que contaran con seguro popular (Prestación que da el Gobierno Federal a 
aquellas personas que no tienen otros medios para tener acceso a seguridad social)

El tipo de muestreo que usamos fue el muestreo teórico, el cual nos permite orientarnos hacia aquellos casos que con base en nuestros intereses pueden aportar más a la explicación del fenómeno, de esta manera se van integrando casos de estudio hasta llegar a la saturación teórica, es decir, el momento en el que ya no surgen nuevas dimensiones o categorías por parte de los participantes con relación al objeto de estudio. A partir de este tipo de muestreo es que se decidió posteriormente a las entrevistas iniciales que la sala de espera es un lugar relevante para el intercambio de información relacionada con la enfer$\operatorname{medad}^{(44,46)}$.

La muestra para las entrevistas quedó compuesta por 19 sujetos, todos creyentes y con seguro popular, 5 hombres, 14 mujeres de una edad promedio de 41,5 años y un ingreso mensual promedio de $\$ 2988.8$ pesos mexicanos. La observación se realizó por 25 días distribuidos en los meses de enero, febrero y agosto de 2008, las observaciones tuvieron una duración promedio de 3 horas por día, para el registro utilizamos diarios de campo y las conversaciones registradas en la sala de espera se transcribieron para su análisis. Todos los usuarios contaban con seguro popular. Esta prestación se da por parte del gobierno federal a aquellas personas que no son derechohabientes de alguna institución de seguridad social, el costo es mínimo y en la mayoría de los casos gratuito para las personas de menos recursos ${ }^{(51)}$.

\section{Procedimiento}

Cuando los sujetos acudían a cita al Departamento de Oncología Pediátrica, se les hacía la invitación a participar en la investigación, posterior a su aceptación se les leía la carta de consentimiento informado redactada acorde a la Declaración de Helsinki y al comité de Enseñanza y Ética del Hospital, y se solicitaba su firma. La duración de la entrevista fue de aproximadamente 1 hora. La observación fue no participante y sólo se transcribieron las conversaciones relacionadas con la temática del cáncer.

\section{RESULTADOS}

En las citas provenientes de entrevistas se coloca entre paréntesis la letra $M$ ó $H$, según se traté de hombre o mujer la persona entrevistada, las letras que le sigue corresponden a las iniciales de los nombres. En los diálogos provenientes de la observación no participante se antecede cada párrafo de acuerdo con la siguiente nomenclatura: MA: Mujer Adulta, HA: Hombre Adulto, MJ: Mujer Joven, HJ: Hombre Joven.

Después de que se identifica en el paciente por parte de los padres algunos indicadores de un posible problema de salud, se inicia la búsqueda del diagnóstico médico. Esta fase implica para los cuidadores primarios y para el paciente un recorrido tanto físico como psicológico; físico porque los medios para obtener un diagnóstico, no se encuentran en su comunidad de origen y deben desplazarse de su comunidad a municipios cercanos y que cuentan con más servicios. El recorrido psicológico se da de pensar en una enfermedad sencilla y no grave a una enfermedad crónica y grave, con posibilidades de morir. Como cualquier viaje o traslado se tienen costos, en este caso el viaje físico obviamente su costo es económico y el viaje o traslado psicológico tiene un costo principalmente emocional. En las siguientes observamos el inicio de este viaje en búsqueda del diagnóstico.

Me Ileve al niño a Villa Juárez y Cerritos. Lo atendieron y le dieron pastillas para el dolor, le hacían análisis y todo, así estuvo 
semana por semana, cada semana le daba el dolor, como unas cuatro o cinco semanas así se la pasó, y luego me lo pasaron para reumatología y le diagnosticaron artritis infantil. Le dieron medicamento para la artritis y como quince días se le calmó el dolor, como un mes todavía se le quitó el dolor, después del mes le volvió, estaba un poquito más amarillo, fue cuando me lo llevé para Cerritos de nuevo y de ahí de Cerritos lo pasaron para acá (San Luis Potosí). Ahí en consulta por urgencias me dijeron que tenía leucemia (M-FAC, ago2007).

Empezó por no querer comer igual que antes, antes andaba bien y después vimos que estaba bajando de peso, lo llevamos con los doctores, le dieron vitaminas y se fue pa abajo y pa abajo hasta que ya se puso muy malo, ya no podía caminar ni hacer nada... en un mes, fue rápido, fue decayendo y decayendo... lo llevé con un doctor que está ahí en San Antonio, es como un hospital, nos dieron medicamentos y no (sirvieron), y luego nos pasaron a Valles y ahí estuve casi una semana y no le hallaban que es lo que tenia y pues ya nos mandaron para acá (M-JCMG, ago2007).

En la búsqueda de atención médica es común que en este ínterin se den diagnósticos erróneos y por tanto medicamento orientado únicamente a paliar los síntomas del paciente. Es común que en las comunidades no se cuente con los recursos estructurales y humanos para realizar el diagnóstico y envíen a los pacientes a una comunidad más grande, sin embargo para tener acceso a medios que permitan un diagnóstico especializado, se requiere tiempo, a veces de meses para realizar un estudio.

Estos traslados provocan una crisis en la familia, específicamente en los roles que desempeñan sus integrantes. Las actividades inherentes al rol de cuidador primario, interfieren con las actividades cotidianas y por tanto con la dinámica familiar que se ha Ilevado hasta entonces. Se debe decidir quién se queda a cargo del paciente $y$ quien se queda para hacerse cargo de los cuidados de la casa y del resto de la familia.

Dice mi hija, tú te vas a trabajar y yo me encargo de él. El otro día cuando me avisaron que estaba enfermo ya estaban aquí en Valles, me vine a Valles y estuve como ocho días con ellos, cuando se vinieron para acá ya no pude venir, porque como le digo yo tenía que estar trabajando para los demás chamacos, no vive su mamá, si viviera como quiera se quedaba con ella, pero ya no vive (H-DHH, mar2008).

Dentro de esta misma crisis se inicia la búsqueda de recursos para obtener la asistencia médica requerida así como para solventar los gastos derivados de la situación de enfermedad, como pago de traslados de su comunidad al hospital, gastos de alimentación y hospedaje derivados de su estancia.

¿Qué hacemos? ¿Dónde conseguimos el dinero?, pues vamos a ver donde, y pos no hay más donde agarrar el dinero, que molestar a las compañeras, compañeros. Anduvimos pensando qué hacemos, porque no tenemos dinero y la trajimos así, no teníamos dinero pero la trajimos aquí a Valles y de ahí nos mandaron acá (a San Luis Potosí). Ahora ya tiene más de un año viniendo. Él paga allá (el presidente municipal) casi mil doscientos gastamos de puro pasaje, nada de digamos que me voy a sentar a comer en el restaurante $o$ a comer algo, no, aquí nosotros como ahorita estamos aguantando hambre porque no tengo dinero. Cuando hay lugar o tiempo nos vamos ahí en el albergue, pero nomás una comida. Dan café también en la mañana a las ocho, pero aquí tenemos que estar a tiempo, presentes, entonces no tomamos café (H-ZSL, ago2007). 
En esta situación surgen otras necesidades de la vida cotidiana, como la atención de salud a otro miembro de la familia. En estos casos se debe decidir en qué se gasta el dinero jerarquizando dichas necesidades, como podemos leer en el siguiente caso:

(una ocasión) se me juntaron las fechas, que tenía que traer a Jesús aquí y tenía que darle yo a ella (hija) para sus estudios que ya estaban programados o sea en el rancho se los programan en el centro de salud a Rio Verde en el hospital general y entonces ¿qué hice yo?... pues le dije a mi hija, ni modo ¿cómo le hago?, no tengo dinero, le digo te vas a esperar $t u$, al niño no puedo dejar que se le pase su tratamiento, nos vamos y me lo traje $y$ paso y ella siguió y siguió traía una bolita aquí también (H-ADM, mar2007).

Esta situación de precariedad promueve las actividades de soporte social enriqueciendo la comunicación entre los sujetos que pasan por la misma situación o situaciones similares y dando pie al afrontamiento colectivo simbólico ${ }^{(52)}$. Las fuentes principales de soporte son la familia y las personas en condiciones similares con quienes se intercambia información de instituciones que pueden proporcionar ayuda para hospedaje, para alimentación, ayuda económica y espiritual.

En estas circunstancias la sala de espera es un lugar importante para el intercambio de información entre las personas involucradas en la enfermedad. La siguiente conversación engloba una de las funciones que tiene la sala de espera, brindar ayuda al otro en la misma situación de enfermedad pero en condiciones más desfavorables.

MJ. Yo siempre le digo a la gente que vayan a mi casa, que se queden ahí, pero nada más me quedan de hablar y no hablan, les doy la dirección y ya ni van.

$$
\text { MA. ¿Sí? }
$$

MJ. ¿Ustedes de dónde son? Le había de decir a su hija, que se vayan a quedar allá

MA. ¿Por dónde vive?

MJ. Ahí por el Saucito, dígale a su hija, le voy a dar la dirección, nada más que la gente anda batallando para pasar por allá por el boulevard que está cerrado.

En parte explica estas conductas de soporte la identificación social de personas que pasan por una situación similar y que tiene conocimiento personal de lo que se vive en tal condición y que dicho conocimiento se vuelve social, compartido entre este grupo, el cual es otra fuente más de información para la construcción de la representación, desde luego estamos hablando de un conocimiento basado en la experiencia persona, contextualizado en contraposición al conocimiento experto, el cual provendría del personal médico .

Esta necesidad constante de recursos para solventar los gastos derivados de la enfermedad, genera también una incertidumbre cuando no se tiene un apoyo ya establecido o acordado para hacer el pago de dichos gastos, de tal forma que esta necesidad económica es otra fuente de ansiedad aparte de la enfermedad en sí.

\section{HA. -Hola ¿Cómo ha estado?}

MA. -Pues bien, echándole ganas.

HA. - ¿Y cómo va con el tratamiento?

MA. - Pues ya más adelantada.

HA. - ¿Y usted está recibiendo ayuda?

MA. -Pues que cree que no, al principio si me ayudaba el DIF, pero ya después me dijeron que ya estaban ayudando a muchos y que hicieron un sorteo para elegir a unos pocos y yo no salí, así que ahorita ando batallando todavía más, pero no puedo hacer nada, más que vivir con todas mis deudas, ya después que se pueda pagar.

HA.- ¿Y quién la está apoyando?

MA.- Pues ahorita ya nadie, le debo un dinero a mi suegra y a mi hermana $y$ 
pues Francisco está trabajando doble turno para sacar un poco más de dinero, pero nada más.

HA.- No pues está bien échele ganas y ojalá termine pronto.

MA.-Y usted cómo va.

HA.- Pues yo ya nada más que tengo ahorita una consulta externa, ya terminé mis radiaciones, eso si me tengo que estar tomando todavía estas pastillitas que están re caras, pero de ahí voy a que me chequen para ver que me dicen.

MA.-A mi todavía me faltan diez radiaciones, pe esto no es tardado.

HA. -No pues no es tardado pero bien que cuesta harto, yo porque a mi si me apoyaron mis hermanos y anduve también en el DIF y en el Centro de salud.

MA. -Pues le digo que a mí también me apoyaba el DIF con mis pasajes, pero ahora ya hasta para eso tengo que andar consiguiendo.

HA-Pues ya ni modo, todo sea por el bien de uno, ya ni que hacerle, si yo también todavía debo cuatro mil pesos, no es que no tenga centavos, pero yo ya les dije que les voy a ir pagando de a poquito y si no que me lleven a la cárcel si quieren, total yo ya estoy curado, bueno, eso digo yo verdad, todavía falta que me chequen a ver qué me dicen.

MA.-Todo sea por uno, como usted dice, a mi todavía me falta poquillo, pero espero terminar pronto, yo a veces pienso, si para está uno cansado dar tantas vueltas, ahora imagínese para los niños (Conversación1, 190208)

La falta de recursos es un elemento determinante en lo que se refiere a la vivencia de la enfermedad, pues tendrá influencia en la búsqueda de medios para la obtención primeramente del diagnóstico a la enfermedad y posteriormente para todo lo que involucra el tratamiento, como el pago de medicamentos y servicios y de aquellas derivadas como son: los traslados al Hospital y el pago de comidas, además influirá también en los efectos psicológicos en el grado de control que la gente experimenta sobre sus vidas. De tal manera que un grupo de pertenencia que mencionan los cuidadores primarios está relacionado con la pobreza.

Me preguntaba ¿Por qué la niña? ¿Por qué nosotros? ¿Por qué casi siempre nos toca a personas que no tenemos solvencia económica para todos estos gastos? (MYLP, feb2008).

A mí me daba mucho coraje y yo decía jay Dios mío! ¿Por qué yo? ¿Por qué habiendo tanta gente rica, por qué yo? porque me decía la doctora, este es un tratamiento muy costoso, y yo no tengo dinero, es muy costoso, muy feo, muy tardado, los tratamientos son de tres años y es muy desgastante para la familia, yo decía, ¡ay, viviendo tan lejos y sin dinero, ¿Por qué yo?, ¿Por qué precisamente yo? (MMMVG, ago2007).

Uno es de escasos recurso económicos, que requieren de todo el apoyo que le puedan brindar y no le brindan nada $(H-$ ADM, mar2007).

El promedio de ingresos de la familia a la que pertenecen los pacientes es de $\$ 2926,47$ pesos mensuales, el cual es obtenido de empleos temporales en oficios como albañilería y agricultura principalmente, de acuerdo al Consejo Nacional de Evaluación de la Política de DesarroIlo Social (CONEVAL) el ingreso neto en promedio, de los hogares en el nivel de pobreza patrimonial es de $\$ 3072,00$, nivel al cual le antecede el de pobreza de capacidades, en esa situación las personas no pueden pagar los productos de la canasta básica y los gastos relacionados con la salud y la educación aún y cuando se asigne el ingreso total sólo a estos rubros $^{(53)}$. 
Como podemos observar las personas involucradas aunque tienen acceso a servicios básicos de salud, la confirmación del diagnóstico de Leucemia implica el trasladarse a otras comunidades y algunas veces pagar servicios particulares, lo que refleja la inequidad al acceso a servicios de salud relacionado con la región en la que se vive ${ }^{(54)}$.

Corrimos con el Doctor, se le inflamó la garganta, nomás que ya tenía tiempo que tenía la bolita y estaba débil... de ahí nos fuimos a Salinas, nos mandaron de Villa de Ramos a Salinas y de ahí nos mandaron aquí, a un consultorio que tienen en el centro médico, ahí fuimos con un Dr. que nos habían recomendado y dijo no es mi especialidad, en aquel edificio amarillo nos recomendó con $X$, llegamos y nos dijo: necesita análisis y salen muy caros, yo trabajo en el Hospital Central mañana ahí los espero, pues si nos venimos otro día, más bien que aquí nos quedamos en un hotelillo y otro día Ilegamos aquí, le hicieron estudios y resultó eso (H-GBA, ago2007).

Fuimos al Centro de Salud de Zacatecas de un rancho que se llama El Capirote y nos dieron vitaminas. No se le quitaba, y luego ya le empezaron a salir moretones en la piel, nos preocupamos más y lo llevamos con el pediatra, pero ya eso fue en Fresnillo y él le mandó hacer estudios y fue donde nos dijo que tenía Leucemia y nos mandó para México, allá estuvimos en México como dos semanas y los Doctores nos preguntaron que de donde éramos y pues ya les dijimos que de Zacatecas y dijeron que porqué nos habíamos ido hasta allá tan lejos, le dije que porque en Zacatecas no había oncólogo y nos dijeron que nos quedaba más cerca San Luis o Aguascalientes y pues ya escogimos aquí (M-MSG, feb2008).
No contar con los recursos para hacer los gastos de tratamiento o diagnóstico genera en el sujeto una disminución en el grado de control que experimentan de sus vidas, ya que esta necesidad, como dijimos anteriormente, se vuelve una fuente más de ansiedad, ya que los cuidadores primarios deben tomar decisiones bajo una gran presión por la urgencia de atención de la enfermedad, de la situación socioeconómica de la familia y de un contexto de incertidumbre respecto a lo que sucede con su paciente; en este momento se debe decidir en qué y cómo gastar el dinero que se tiene, así como establecer los roles de cuidador primario y por tanto de quién se queda en casa a cargo del resto de la familia y de su atención. Esta característica como mencionamos en líneas anteriores es indicador del nivel pobreza de capacidades cuyas características destacan que hay insuficiencia del ingreso para efectuar los gastos necesarios en salud ${ }^{(53)}$.

\section{DISCUSIÓN}

Los resultados obtenidos en esta investigación nos permiten confirmar la influencia que tiene el contexto en la construcción de la experiencia de enfermedad en los padres-cuidadores primarios y sobre todo la ansiedad que se genera a partir de la carencia de recursos.

Las carencias económicas son fuente de sufrimiento ante la imposibilidad de pagar todos los servicios y gastos médicos que la enfermedad del paciente requie$\mathrm{re}^{(55,56)}$. Dadas esas condiciones el grupo desarrolla estrategias para salir delante de esa situación las cuales se comparten con aquellos considerados endogrupo, como parte de la solidaridad intragrupal.

La pobreza y la enfermedad en el marco del contexto socio cultural, son fuente para anclar el fenómeno nuevo, en este caso la leucemia, por tanto el abordaje que se debe dar para apoyar a los cui- 
dadores primarios debe considerar estas características, en acciones tales como la comunicación del diagnóstico, el uso de tratamientos alternativos y actividades religiosas como forma de explicarse y afrontar la situación.

La pobreza no sólo es fuente de estrés para el cuidador primario, también es causa de una salud pobre ${ }^{(56)}$. En un contexto de carencias es común que no se tenga acceso a bienes como agua potable, servicio de drenaje, clínicas equipadas con recursos humanos y físico, entre otros, por tanto se reducen las posibilidades de acceso a vacunas a una alimentación adecuada, o a servicios médicos especializados como ya hemos visto. De tal suerte que se conforma un ciclo que confirma la fuerte correlación entre vivir en la pobreza y tener una salud pobre ${ }^{(57)}$.

De acuerdo a Organismos internacionales como la Organización de las Naciones Unidas alcanzar mejores niveles de salud requiere reducir los índices de pobreza $^{(54)}$. De tal forma que los gobiernos en sus políticas sociales deberían considerar esta relación. De forma más concreta quienes intervenimos en el área de la salud no podemos dejar de considerar que una intervención de trabajo en salud en zonas de pobreza debe ser una intervención integral, es decir, una intervención biopsicosocial orientada al desarrollo comunitario.

\section{REFERENCIAS BIBLIOGRÁFICAS}

1. World Health Organization. World Health Statistics 2006. France: World Health Organization 2006.

2. World Health Organization. World health statistics 2010. France: World Health Organization 2010.

3. Stiller C, Quinn M, Rowan S. Childhood Cancer. The health of children and young people. Great Britain: Office of National Statistics 2004:3-19.

4. Instituto Nacional de Estadística y Geografía. Estadísticas a propósito del día mun- dial del cáncer. México: Instituto Nacional de Estadística y Geografía; 2008.

5. Instituto Nacional de Estadística Geografía e Informática. Estadísticas Vitales de San Luís Potosí. Cuaderno Núm. 8. Edición 2005. México: Instituto Nacional de Estadística Geografía e Informática 2006.

6. Secretaría de Salubridad y Asistencia. Principales causas de mortalidad en edad escolar 5 a 14 años. 2005 [Acceso 02 de octubre de 2007; Disponible en: http://sinais.salud.gob.mx/mortalidad/

7. National Cancer Institute. Young People With Cancer. A Handbook for parents. United States of America: U.S. Department of Health and Human Services, Public Health Service, National Institutes of Health 2001.

8. El Banco Mundial. Gasto en salud per cápita (US\$ a precios actuales) 2011 [Acceso 201122 Octubre 2011]; Disponible en: http://datos.bancomundial.org/indicador/ SH.XPD.PCAP

9. Senado de la República LX Legislatura. Dictamen con punto de acuerdo por el que se exhorta al titular del poder ejecutivo federal a fortalecer las medidas para atender y prevenir el cáncer infantil. Gaceta del Senado. 2008;5.

10. Pérez P, F. Respuestas emocionales, enfermedad crónica y familia. En: FernándezAbascal, Palmero F, editores. Emociones y salud. Barcelona: Ariel 1999p.301-324.

11. Almanza-Muñoz J, Holland J. Psicooncología: estado actual y perspectivas futuras. Rev Inst Nal Cancerol (Mex). 2000;46(3):196-206.

12. Arrivillaga QM, Correa SD, Salazar TI. Psicología de la salud: abordaje integral de la enfermedad crónica. Bogotá: El Manual Moderno 2007.

13. Wheis K, Reiss D. Family reorganization in response to cancer: A developmental perspective. In: Baider L, Cooper C, Kaplan De-Nour A, editores. Cancer and the family. Chichester, England: John Wiley and Sons, 2000. p.17-40. 
14. Bloom JR. The role of family support in cancer control. In: Baider L, Cooper CL, Kaplan De-Nour A., editors. Cancer and the family. 2 ed. England: John Wiley \& Sons Ltd. 2000. p.55-72.

15. Moral de la Rubia J, Martínez R, Juan. Estrés y afrontamiento de los padres frente al diagnóstico de cáncer del hijo. En: Álvarez B, Javier, Moral de la Rubia J, Martínez R, Juan, editores. Psicología e investigación en enfermedades degenerativas crónicas. México: Universidad Autónoma de Nuevo León 2009. p. 41-101.

16. Robles SL. La invisibilidad del cuidado de los enfermos crónicos: Un estudio cualitativo en el barrio de Oblatos. Guadalajara: Editorial Universitaria 2007.

17. Woznick L, Goodheart C. Living with childhood cancer. Washington, DC: American Psychological Association 2002.

18. Barbara M, Kaplan B, Fogel J. Childhood health issues across the life span. In: Baum A, Revenson T, Singer J, editores. Handbook of health psychology. New Jersey: Lawrenece Erlbaum Associates 2001. p. 449-56.

19. Barrera M, Fleming C, Khan F. The role of emotional social support in the psychological adjustment of siblings of children with cancer. Child Care Health Dev 2004 30(2):103-11. Doi: 10.1111/j.1365-2214.2003.00396.x

20. Laurel LN. Helping families of patients with cancer. Oncol Nurs Forum 2005;32(4):74350. Doi: 10.1177/1043454206293266

21. Patterson J, Holm K, Gurney J. The impact of childhood cancer on the family: A qualitative analysis of strains, resources, and coping behaviours. Psychooncology 2004 13:390-407. Doi: 10.1002/pon.761

22. Barrera $M, D^{\prime}$ Agostino NM, Gibson J, Gilbert T, Weksberg R, Malkin D. Predictors and mediators of psychological adjustment in mothers of children newly diagnosed with cancer. Psychooncology 2004;13:630 -41. Doi: 10.1002/pon.3078

23. Ow R. Burden of care and childhood can- cer: experiences of parents in an Asian context. Health Soc work 2003;28(3):23240. Doi:10.1093/hsw/28.3.232

24. Bashore L, Smith A. The Effect of clinicbased health promotion education on perceived health status and health promotion behaviors of adolescent and young adult cancer survivors. J Pediatr Oncol Nurs 2006;23(6):326-34. Doi: 10.1177/1043454206293266

25. Krueger G. Hope and suffering: Children, cancer, and the paradox of experimental medicine. Baltimore: Johns Hopkins University Press 2008.

26. Taïeb O, Moro MR, Baubet T, Revah-Lévy A, Flament M. Posttraumatic stress symptoms after childhood cancer Eur Child Adolesc Psychiatry. 2003;12(6). Doi: 10.1007/s00787-003-0352-0

27. Coffey JS. Parenting a child with chronic illness: A metasynthesis. Pediatr Nurs 2006;32(1):51-9.

28. Di Gallo A. While my sister went to the disco, I went to hospital and met the doctors: Narrative as a measure of the psychological integration of the experience of cancer in childhood and adolescence. Clin Child Psychol Psychiatry 2003;8(4):489-502. Doi:10.1177/13591045030084006

29. Gedaly-Duff V, Lee KA, Nail LM, Nicholson S, Johnson KP. Pain, sleep disturbance, and fatigue in children with leukemia and their parents: A pilot study. Oncol Nurs Forum 2006;33(3):641-6. Doi:10.1188/06. ONF.641-646

30. Eiser C, Eiser JR, Stride CB. Quality of life in children newly diagnosed with cancer and their mothers. Health Qual Life Outcomes 2005:3:29. Doi: 10.1186/14777525-3-29

31. Svavarsdottir EK. Surviving childhood cancer: Parents'perceptions of their child's health. J Pediatr Oncol Nurs 2005;22(2):808. Doi:10.1177/1043454204273812

32. Moscovici S. Razón y culturas. Discurso pronunciado con motivo de la investidura como Doctor "Honoris Causa" por la Uni- 
versidad de Sevilla por el Profesor Serge Moscovici. En: Universitaria UdSE, editor. Acto Solemne de Investidura como Doctor Honoris Causa del Excmo Sr Dr D Serge Moscovici. Sevilla: Universidad de Sevilla 1993:45-94.

33. Moscovici S. Notes towards a description of social representations. Eur J Soc Psychol 1988;18:211-50.

34. Moscovici S, Marková I. Presenting social representations: A conversation. Cult Psychol 1998;4(3):371-410. Doi:10.1177/1354067X9800400305

35. Wagner W, Hayes N. Everyday Discourse and common-sense: The theory of social representation. New York: Palgrave McmiIlan 2005.

36. Jodelete D. Conferencia de la Dra. Denise Jodelet. En: Aires UdB, editor. Primeras Jornadas sobre Representaciones Sociales; 2003; Buenos Aires, Argentina: Universidad de Buenos Aires, 2003.

37. Moscovici S. The phenomenon of social representations. En: Farr R, Moscovici S, editores. Social representations. Cambridge, Cambridge, Paris: Editions de la Maison des Sciences de I'Homme. p.3-69

38. Jodelet D. La representación social: Fenómenos, conceptos y teoría. En: Moscovici S, editor. Psicología Social. España: Ediciones Paidós Ibérica, S. A. 1986:469-94.

39. Moscovici S. La psychanalyse son image et son public. 2a ed. France: Presses Universitaires de France 1976.

40. Wagner W. Fields of research and sociogenesis of social representations: A discussion of criteria and diagnostics. Soc Sci Inform. 1994;33(2):199-228.

41. Flick U. Social representations of health held by health professionals: The case of general practitioners and home-care nurses. Soc Sci Inform 2002;41(4):581-602. Doi: 10.1177/053901898037004005

42. Flick $U$. Delightful representations of the paradoxes in the social world: Discussion of M. J. Spink's paper. Papers on Social Representations. 1993;2(1):60-5.
43. Flick U. Introducción a la investigación cualitativa. 1a ed. Madrid: Morata 2004.

44. Flick U. Qualitative research-state of the art. Soc Sci Inform 2002;41(1):5-24. Doi: 10.1177/0539018402041001001

45. Strauss A, Corbin J. Basics of qualitative research. Techniques and procedures for developing Grounded Theory. 2 ed. United States of America: Sage Publications, Inc. 1998.

46. Kohlbacher F. The Use of Qualitative content analysis in case study research. Forum: Qual Soc Res 2006;7(1).

47. Mayring P. Qualitative Content Analysis Forum Qualitative Sozialforschung / Forum: Qual Soc Res 2000:28 paragraphs.

48. Krippendorff K. Content analysis: An introduction to its methodology. 2 ed. United States of America: Thousand Oaks, CA: Sage. 2004.

49. Elo S, Kyngäs $H$. The qualitative content analysis process. J Adv Nurs 2008;62(1):107-15. Doi: 10.1111/j.13652648.2007.04569.x

50. Silverman D. Interpreting qualitative data. Methods for analysing talk, text and interaction. 2 ed. London, Thousand Oaks, New Delhi: Sage Publications 2001.

51. Centro de Estudios Sociales y de Opinión Pública, Cámara de Diputados LIX Legislatura. Programa Seguro Popular. 2005 [Acceso 2010] Disponible en: http://www. diputados.gob.mx/cesop/doctos/El\%20Seguro\%20Popular.pdf

52. Wagner W, Duveen G, Farr R, Jovchelovitch S, Lorenzi-Cioldi F, Marková I, et al. Theory and method of social representations. Asian J Soc Psychol 1999;2:95-125.

53. Consejo Nacional de Evaluación de la Política de Desarrollo Social. Informe Ejecutivo de Pobreza México 2007. Informe Ejecutivo para el Diagnóstico del Plan Nacional de Desarrollo 2007-2012. México: Consejo Nacional de Evaluación de la Política de Desarrollo Social 2007 060309.

54. Muhutdinova R. World Health Organiza- 
tion. Encyclopedia of World Poverty 2006 [Acceso 10 marzo 2009; Disponible en: http://www.sage-ereference.com/worldpoverty/Article_n765.html? searchQuery=y\% 3D17\%26quickSearch\%3DMuhutdinova $\% 26 x \% 3 D 38$

55. Dailey AB. Poverty and Health. Encyclopedia of Epidemiology 2007 [Acceso 10 de enero de 2008]; Disponible en: http:// sage-ereference.com/ epidemiology/Article_n361.html
56. Dynan L. Healthcare. Encyclopedia of World Poverty 2006 [Acceso el 10 de marzo de 2009]; Disponible en: http:// sage-ereference.com/ worldpoverty/Article_n314.html

57. Suarez-Balcazar Y, Cooper S. Poverty. En: Albrecht G, editor. Encyclopedia of Disability: Sage publications 2005.p. 1281-4 